University of Nebraska - Lincoln

DigitalCommons@University of Nebraska - Lincoln

$7-1-2006$

\title{
Adsorption of hydrogen molecules on the platinum-doped boron nitride nanotubes
}

Xiaojun $\mathrm{Wu}$

University of Nebraska-Lincoln, jwu4@unl.edu

J.L. Yang

Hefei National Laboratory for Physical Science at Microscale, University of Science and Technology of China, Hefei 230026, People's Republic of China

Xiao Cheng Zeng

University of Nebraska-Lincoln, xzeng1@unl.edu

Follow this and additional works at: https://digitalcommons.unl.edu/chemzeng

Part of the Chemistry Commons

Wu, Xiaojun; Yang, J.L.; and Zeng, Xiao Cheng, "Adsorption of hydrogen molecules on the platinum-doped boron nitride nanotubes" (2006). Xiao Cheng Zeng Publications. 6.

https://digitalcommons.unl.edu/chemzeng/6

This Article is brought to you for free and open access by the Published Research - Department of Chemistry at DigitalCommons@University of Nebraska - Lincoln. It has been accepted for inclusion in Xiao Cheng Zeng Publications by an authorized administrator of DigitalCommons@University of Nebraska - Lincoln. 


\title{
Adsorption of hydrogen molecules on the platinum-doped boron nitride nanotubes
}

\author{
Xiaojun $\mathrm{Wu}$ \\ Department of Chemistry, University of Nebraska-Lincoln, Lincoln, Nebraska 68588 and \\ Center for Materials Research and Analysis, University of Nebraska-Lincoln, Lincoln, Nebraska 68588 \\ J. L. Yang \\ Hefei National Laboratory for Physical Science at Microscale, University of Science and Technology \\ of China, Hefei 230026, People's Republic of China and Department of Chemical Physics, University \\ of Science and Technology of China, Hefei 230026, People's Republic of China \\ X. C. Zeng ${ }^{\text {a) }}$ \\ Department of Chemistry, University of Nebraska-Lincoln, Lincoln, Nebraska 68588 and \\ Center for Materials Research and Analysis, University of Nebraska-Lincoln, Lincoln, Nebraska 68588
}

(Received 29 December 2005; accepted 12 May 2006; published online 26 July 2006)

\begin{abstract}
Adsorption of hydrogen molecules on platinum-doped single-walled zigzag $(8,0)$ boron nitride $(\mathrm{BN})$ nanotube is investigated using the density-functional theory. The Pt atom tends to occupy the axial bridge site of the $\mathrm{BN}$ tube with the highest binding energy of $-0.91 \mathrm{eV}$. Upon Pt doping, several occupied and unoccupied impurity states are induced, which reduces the band gap of the pristine BN nanotube. Upon hydrogen adsorption on Pt-doped BN nanotube, the first hydrogen molecule can be chemically adsorbed on the Pt-doped $\mathrm{BN}$ nanotube without crossing any energy barrier, whereas the second hydrogen molecule has to overcome a small energy barrier of $0.019 \mathrm{eV}$. At least up to two hydrogen molecules can be chemically adsorbed on a single Pt atom supported by the BN nanotube, with the average adsorption energy of $-0.365 \mathrm{eV}$. Upon hydrogen adsorption on a Pt-dimer-doped $\mathrm{BN}$ nanotube, the formation of the Pt dimer not only weakens the interaction between the Pt cluster and the BN nanotube but also reduces the average adsorption energy of hydrogen molecules. These calculation results can be useful in the assessment of metal-doped BN nanotubes as potential hydrogen storage media. () 2006 American Institute of Physics. [DOI: 10.1063/1.2210933]
\end{abstract}

\section{INTRODUCTION}

Hydrogen has attracted enormous research attention as a future energy resource. However, wide application of hydrogen as fuel requires that the storage of hydrogen can be processed more economically and conveniently than the state of the art. Recently, increasing attention has been paid to the carbon nanotube as a potential hydrogen storage medium due to its light mass density and high surface to volume ratio, as well as high degrees of reactivity between the carbon and hydrogen. ${ }^{1-4}$ However, experimental results reported from different groups have been controversial particularly on the capacity of hydrogen storage in single-walled carbon nanotubes (SWCNTs). ${ }^{5}$ One possible reason for this controversy is that SWCNTs can be either semiconductor or metal, depending on their diameters and helicities. As such, SWCNTs synthesized by different groups may be quite different in the electronic properties due to certain differences in the tube diameter and helicity. ${ }^{6}$ Boron nitride (BN) nanotubes, which are analog to carbon nanotubes in many aspects, are widegap semiconductor with their band gap only weakly dependent on the tube diameter, helicity, and the number of tube walls. Additionally, BN nanotubes are chemically and thermally more stable. ${ }^{7-10}$ Given these unique properties of BN nanotubes, some efforts have been made recently to assess

${ }^{a)}$ Electronic mail: xczeng@phase2.unl.edu
BN nanotubes as a potential hydrogen storage medium. ${ }^{11,12}$ Ma et al. reported that the multiwalled and bamboolike BN nanotubes can adsorb hydrogen up to $2.6 \mathrm{wt} \%$ wherein $70 \%$ of the hydrogen is chemisorbed. ${ }^{11}$ Tang et al. found that hydrogen storage can even reach to $4.2 \mathrm{wt} \%$ if the $\mathrm{BN}$ nanotubes were treated with $\mathrm{Pt}^{12}$ These experimental measurements suggest that metal-doped BN nanotubes may hold some potential as hydrogen storage media.

Some theoretical efforts have also been made to assess hydrogen-adsorption properties of $\mathrm{BN}$ nanomaterials. ${ }^{13-18}$ We recently studied the adsorption of hydrogen atoms on BN nanotubes. ${ }^{13,14}$ Our previous density-functional theory (DFT) calculations show that hydrogen storage up to $4.0 \mathrm{wt} \%$ may be feasible via hydrogen-atom adsorption, which corresponds to $50 \%$ coverage of the surface of BN nanotubes. However, the calculated average adsorption energy was found to be less than the covalent bond energy of hydrogen molecule. Jhi and Kwon studied the physisorption of hydrogen molecule on BN nanotubes. They showed that the adsorption energy is higher than that on carbon nanotubes. ${ }^{15}$ Han et al. studied the collision of hydrogen molecule with single-walled BN nanotubes. ${ }^{16}$ They observed that hydrogen molecules can enter into the tube through the hexagon ring of the sidewall, even though the incident energy of the hydrogen molecule is very high (the activation energy of chemical dissociation of a hydrogen molecule on the pristine 
sidewall can be as high as $130 \mathrm{kcal} / \mathrm{mol}$ ). These previous theoretical works also suggested that $\mathrm{BN}$ nanotubes might hold some potential as hydrogen storage media. However, the experimental result of the high percentage $(70 \%)$ of chemisorbed hydrogen has not yet been explained. ${ }^{11}$ In another study, we examined the adsorption and chemical dissociation of hydrogen molecule on defected BN nanotubes. ${ }^{17}$ With defects, the chemical dissociation of hydrogen molecule becomes exothermic and the energy barrier of dissociation can be appreciably reduced, which may partially account for the high percentage of hydrogen chemisorption on the BN nanotubes. Although the activation energy of hydrogen dissociation can be reduced by the defects, the desorption energy of the hydrogen is still very high, which limits the real-world application of BN nanotubes for hydrogen storage. More recently, hydrogen adsorption on metal-doped carbon nanotubes and fullerenes has been investigated. ${ }^{19-23}$ The doping of SWCNT and fullerenes can generally promote more hydrogen uptake. The aim of this article is to assess to what extent the Pt-doped BN nanotubes can enhance the hydrogen uptake by using DFT methods. With one or two Pt atoms doped on the outer surface of the $\mathrm{BN}$ nanotube, the hydrogen-atom adsorption, hydrogen-molecule adsorption/ dissociation, and the hydrogen storage capacity are computed. We found that hydrogen molecules can be chemically adsorbed on the Pt-doped BN nanotubes fairly easily. The calculation results offer an explanation to the enhanced percentage of hydrogen chemisorption observed in the experiment.

\section{MODEL SYSTEM AND COMPUTATIONAL METHODS}

The calculations were carried out by using the densityfunctional method implemented in the DMOL3 package. ${ }^{24}$ Allelectron calculations were undertaken with the double numerical plus polarization (DNP) basis sets and the generalized-gradient approximation with the Perdew-BurkeErnzerhof (PBE) functional. ${ }^{25}$ The real-space global cutoff radius of all atoms was set to be $5.5 \AA$. Spin-unrestricted DFT was used to obtain all the results reported below. For simplicity, we chose a single-walled zigzag $(8,0)$ BN nanotube as the model system. A tetragonal supercell of the size $20 \times 20 \times 8.64 \AA^{3}$ with the length of $c$ equal to twice the periodicity of the $(8,0) \mathrm{BN}$ nanotube is adopted. The tube axis is set along the $z$ direction. The Brillouin zone is sampled by $1 \times 1 \times 3$ special $k$ point using the MonkhorstPack scheme. ${ }^{26}$ Test calculations showed that increasing the $k$ points has little change in the final results.

\section{RESULTS AND DISCUSSION}

First, we examined the adsorption site on which a $\mathrm{Pt}$ atom can give rise to the highest adsorption energy on the single-walled $(8,0) \mathrm{BN}$ nanotube. Five different sites were tested, including the top site of the boron atom (B), the top site of the nitrogen atom $(\mathrm{N})$, the hollow site of the hexagon $\mathrm{BN}$ ring $(\mathrm{H})$, the bridge site over an axial $\mathrm{BN}$ bond (BA), and the bridge site over a zigzag $\mathrm{BN}$ bond (BZ), as shown in Fig. 1(a). After full structural optimization, the Pt atom al- (a)

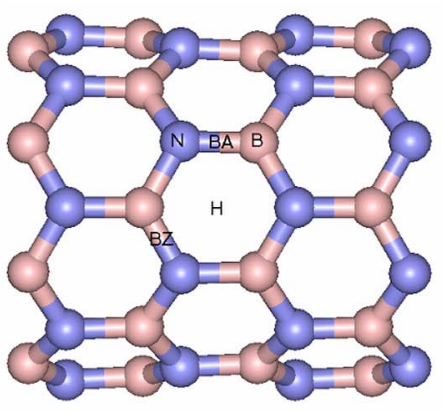

(b)

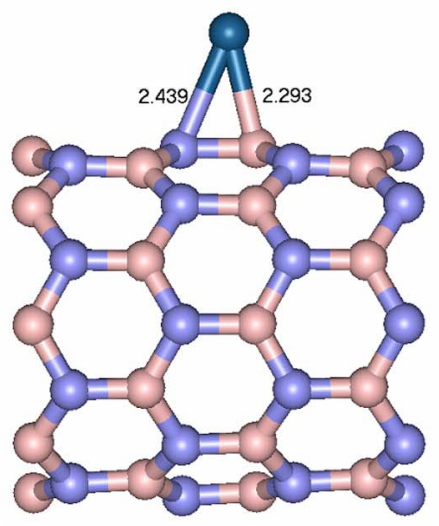

(c)

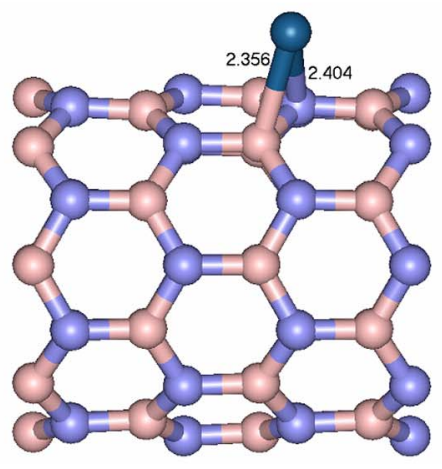

FIG. 1. (Color online) (a) The optimized geometric structures of a pristine single-walled zigzag $(8,0) \mathrm{BN}$ nanotube. The optimized structures of the $\mathrm{Pt}$-doped BN nanotube with the Pt atom at the (b) BA site or (c) BZ site.

ways locates on either the BA or BZ site [Fig. 1(b)], regardless of the initial location. It turns out that the Pt atom is more stable on the BA site than on the BZ site. The calculated binding energy is $-0.91 \mathrm{eV}$ at the BA site, on which the lengths for the $\mathrm{Pt}-\mathrm{B}$ and $\mathrm{Pt}-\mathrm{N}$ bonds are 2.44 and $2.29 \AA$, respectively. At the $\mathrm{BZ}$ site, the corresponding bond lengths are nearly the same as those at the BA site. Although the Pt atom has an unfilled $d$ orbital, the spin-charge analysis shows that there is no net spin charge on any atoms of the Pt-doped $\mathrm{BN}$ nanotube.

Next, with the Pt atom doped at the BA site, we examined the hydrogen-molecule adsorption on the Pt-doped BN nanotube. We placed one hydrogen molecule near the $\mathrm{BN}$ nanotube. This very first hydrogen molecule can be chemically adsorbed on the Pt atom without crossing any energy 


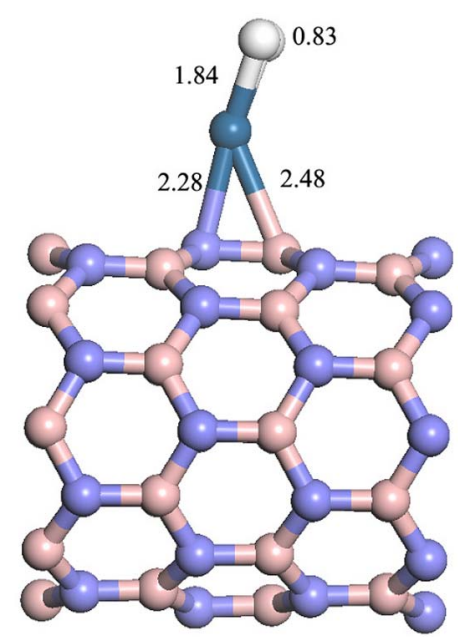

FIG. 2. (Color online) The optimized geometric structure of a $\mathrm{H}_{2}$ adsorbed on the Pt-doped $(8,0) \mathrm{BN}$ nanotube, where the Pt atom is located at the BA site.

barrier. Eventually, the hydrogen molecule is partially dissociated with the $\mathrm{H}-\mathrm{H}$ bond length being $0.83 \AA$ (Fig. 2). The calculated adsorption energy, defined as

$$
\begin{aligned}
E_{\mathrm{ads}}= & E\left(\mathrm{BNNT}+\mathrm{Pt}+\mathrm{H}_{n+2}\right) \\
& -E\left(\mathrm{BNNH}+\mathrm{Pt}+\mathrm{H}_{n}\right)-E\left(\mathrm{H}_{2}\right),
\end{aligned}
$$

is $-0.62 \mathrm{eV}$. Here, the minus adsorption energy denotes exothermic adsorption. The two $\mathrm{H}-\mathrm{Pt}$ bond lengths are $1.84 \AA$; the $\mathrm{Pt}-\mathrm{B}$ bond length is $2.48 \AA$ and $\mathrm{Pt}-\mathrm{N}$ bond length is $2.28 \AA$. Compared to the Pt-BN tube structure in absence of the hydrogen molecule, the adsorption of the hydrogen molecule actually weakens the Pt-B bond but slightly strengthens the Pt-N bond. We note that this hydrogen-adsorption behavior differs from that reported previously ${ }^{20}$ for Ti-doped carbon nanotube, on which the first hydrogen molecule can dissociate spontaneously near the $\mathrm{Ti}$ atom. This difference in hydrogen adsorption can be attributed to the difference in the location of the metal dopant, that is, in the Ti-doped carbon nanotube, the Ti atom tends to occupy the hollow site of the carbon nanotube whereas in the Pt-doped BN nanotube the $\mathrm{Pt}$ atom favors the BA site. Indeed, it has been reported that for the Pt-doped carbon nanotube, ${ }^{22}$ the first $\mathrm{H}_{2}$ is actually adsorbed in the molecular form if the $\mathrm{Pt}$ atom occupies the bridge site of the carbon nanotube. On the other hand, we also obtained a locally stable configuration such that the adsorbed hydrogen is in the atomic form. However, the binding energy is less compared to that when the $\mathrm{H}_{2}$ is adsorbed in molecular form.

Furthermore, we considered the addition of the second hydrogen molecule to the system. As the second hydrogen molecule approaches the Pt-doped BN nanotube, initially, it is physically adsorbed onto the Pt atom with small adsorption energy of $-0.023 \mathrm{eV}$, as shown in Fig. 3(a). The H-Pt distance is about $2.24 \AA$ while the $\mathrm{H}-\mathrm{H}$ bond length is about $0.77 \AA$. After crossing an energy barrier, the second hydrogen molecule can be chemically adsorbed onto the Pt-doped BN nanotube [Fig. 3(b)] with net increased adsorption energy of $-0.11 \mathrm{eV}$ (relative to the most stable configuration (a)

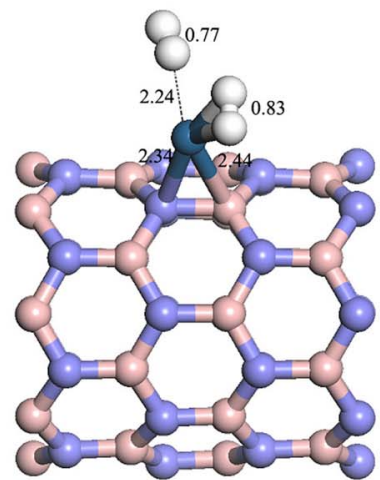

(b)

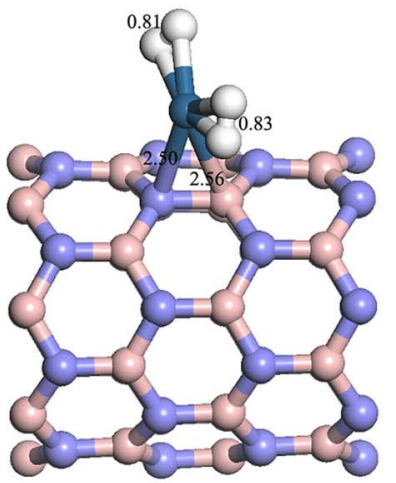

(c)

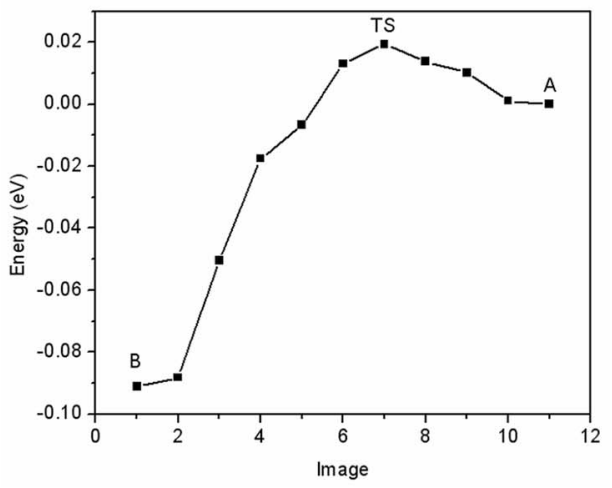

FIG. 3. (Color online) The optimized geometric structures of two hydrogen molecules adsorbed on the Pt-doped $(8,0) \mathrm{BN}$ nanotube in the case of (a) physical adsorption and (b) chemical adsorption. (c) The calculated MEP connecting the physical adsorption state and the chemical adsorption state.

with the first hydrogen molecule adsorbed). Thus, the averaged adsorption energy per hydrogen molecule is $-0.365 \mathrm{eV}$. Upon the adsorption of the second $\mathrm{H}_{2}$, the Pt-B and Pt-N bonds are, respectively, elongated to 2.56 and $2.50 \AA$, while the $\mathrm{H}-\mathrm{H}$ bond length of the second hydrogen molecule is elongated to $0.81 \AA$. It is clear that the second (weakly chemisorbed) hydrogen molecule further weakens the interaction between the $\mathrm{Pt}$ atom and the $\mathrm{BN}$ nanotube. To determine the energy barrier to the adsorption of the second hydrogen molecule, we calculated the minimum-energy path (MEP) for the adsorption process with the nudged elastic 
(a)

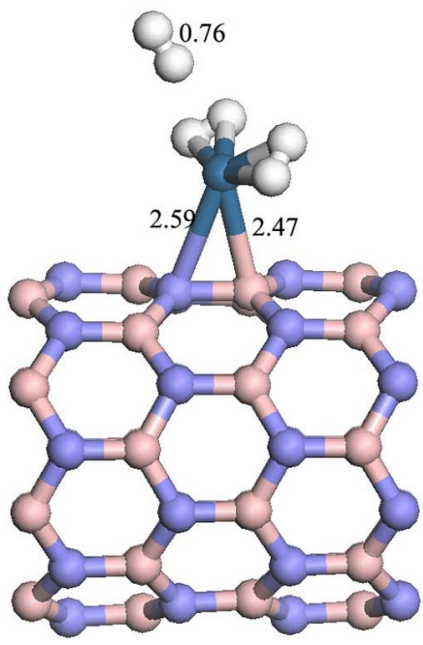

(b)

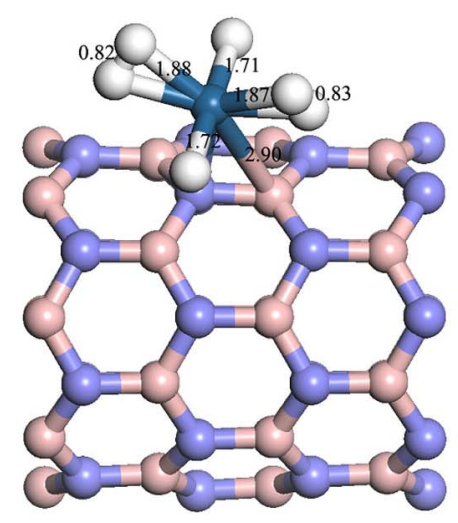

FIG. 4. (Color online) Two possible geometries for three hydrogen molecules adsorbed on the Pt-doped BN nanotube. The geometry in (a) is energetically more favorable than that in (b).

band (NEB) method implemented in DMOL3 package. ${ }^{27}$ The physisorption structure is set as the initial state [A in Fig. $3(\mathrm{c})]$ and the chemisorption structure as the final state $[\mathrm{B}$ in Fig. 3(c)]. As shown in Fig. 3(c), the MEP shows that the energy barrier is merely $0.019 \mathrm{eV}$, indicating that the chemisorption of the second hydrogen molecule on the Pt-doped $\mathrm{BN}$ nanotube can proceed quite easily.

To determine the maximum number of hydrogen molecules that can be chemically adsorbed on the Pt-doped BN nanotube, we examined the addition of the third hydrogen molecule $\left(\mathrm{H}_{2}\right)$ to the system. We placed the third $\mathrm{H}_{2}$ at several different initial locations near the $\mathrm{Pt}$ atom. We found that regardless of the initial location, the third $\mathrm{H}_{2}$ tends to move away from the Pt atom and cannot be chemically adsorbed. The optimized structure in Fig. 4(a) shows that the average distance between the center of the third $\mathrm{H}_{2}$ and the $\mathrm{Pt}$ atom is about $2.37 \AA$. The corresponding adsorption energy is about $-0.02 \mathrm{eV}$ which stems mainly from the van de Waals interaction. This result indicates that up to two hydrogen molecules can be chemically adsorbed on a single Pt atom supported by the BN nanotube. Interestingly, when we placed hydrogen atoms instead of molecules onto the Pt atom, we found that up to six hydrogen atoms can be chemically ad-

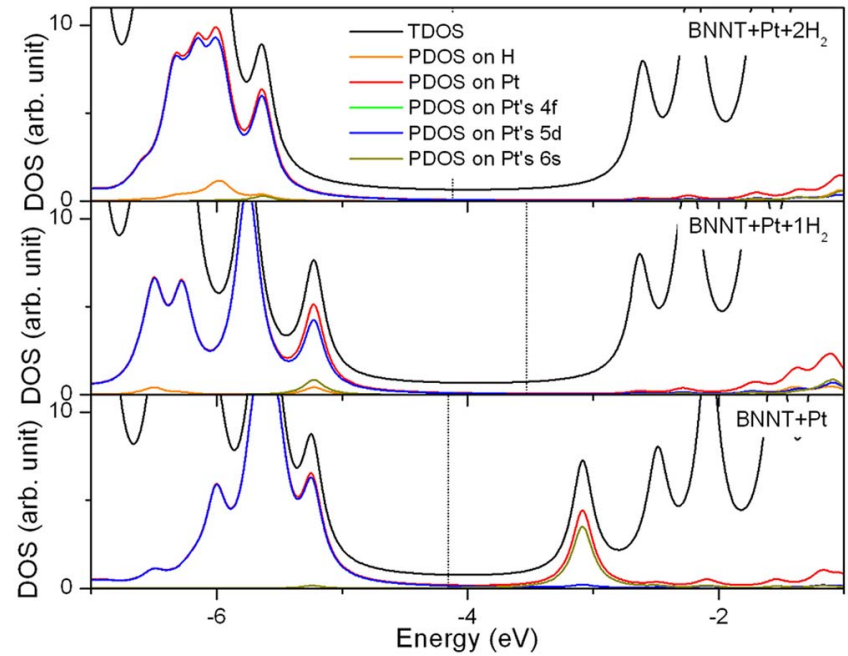

FIG. 5. (Color online) The DOS of a pristine BN nanotube, a Pt-doped BN nanotube with and without hydrogen adsorption. The total DOS is projected onto the atomic orbitals of $\mathrm{Pt}$ and $\mathrm{H}$ atoms.

sorbed on a single Pt atom, as shown in Fig. 4(b). However, this configuration is less stable by $1.19 \mathrm{eV}$ than the molecular configuration shown in Fig. 4(a). After we placed the eighth hydrogen atoms near the $\mathrm{Pt}$ atom, we found that the $\mathrm{Pt}$ atom was detached from the BN nanotube, which suggests a possible way to remove (clean) the Pt dopant from the BN nanotube.

We also calculated the band structure of pristine BN nanotube as well as that of the Pt-doped BN nanotube, with and without hydrogen-molecule adsorption. The band structures show that the doping of a Pt atom per supercell induces six impurity states inside the band gap of the pristine BN nanotube, one of which is unoccupied while the other five are occupied. The band gap is reduced from 3.65 to $2.15 \mathrm{eV}$. With the adsorption of the first hydrogen molecule, the unoccupied impurity state disappears, while the occupied impurity states are nearly unchanged. In Fig. 5, we plot the calculated density of states (DOS) and projected DOS on the Pt atom and hydrogen molecules. It can be seen that the unoccupied impurity state mainly stems from the Pt's $s$ orbital, while the occupied impurity states are dominated by the Pt's $d$ orbitals. Moreover, the adsorption of the first hydrogen molecule is due to the interaction between the $s$ orbital of Pt atom and hydrogen molecule. Note also that this electronic-structure behavior is somewhat different from that of metal-doped carbon nanotube. Here, it is the unoccupied impurity state that interacts with the hydrogen molecule as the first hydrogen molecule is chemically adsorbed.

Using the Hirshfeld method, we found that about $0.12 e$ charge is transferred from the Pt atom to the $\mathrm{BN}$ nanotube when the Pt atom is adsorbed at the BA site. This chargetransfer behavior bears some resemblance to the case of metal-doped carbon nanotube or $\mathrm{C}_{60}$, in which the charge is also transferred from the metal dopant to the carbon nanotube or $\mathrm{C}_{60}$. The charge transfer leaves the metal dopant in cationic form so that the hydrogen molecule can be trapped by the metal cation via the charge polarization mechanism. ${ }^{23}$ Assuming the same mechanism is applicable to the case of 
(a)

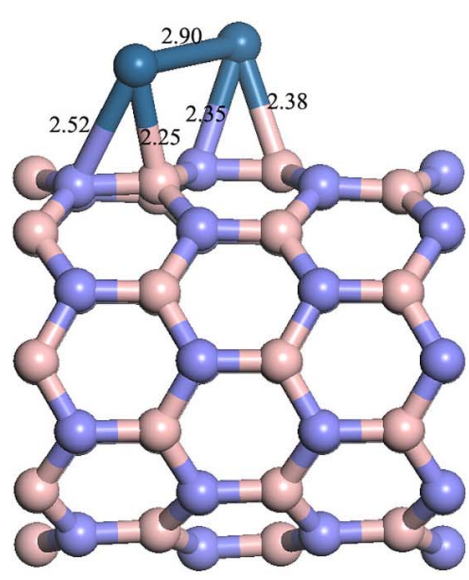

(b)

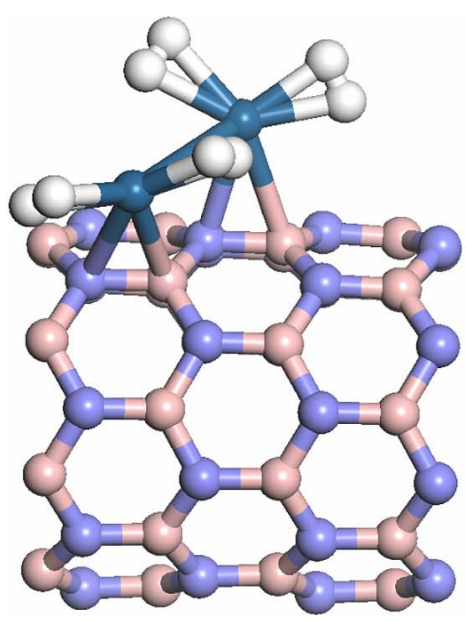

FIG. 6. (Color online) (a) The optimized geometric structure of BN tube doped with a Pt dimer. (b) The optimized structure of four hydrogen molecules adsorbed on the Pt-dimer-doped BN nanotube.

BN nanotube, the decrease of the adsorption energy with increasing the number of $\mathrm{H}_{2}$ can be understood: When more than one hydrogen molecule are adsorbed on the Pt-doped $\mathrm{BN}$ tube, there is effectively less charge transfer from the Pt-doped BN nanotube to every hydrogen molecules. Besides the charge-transfer mechanism, chemical interaction may also play an important role in the adsorption process due to the charge overlap.

Third, we examined the BN nanotube doped with a small $\mathrm{Pt}$ cluster, e.g., a Pt dimer. Specifically, the first Pt atom is placed at the BA site of the BN nanotube while the second Pt atom can be initially placed at various locations near the first Pt atom. After full geometry optimization with various locations of the second Pt atom, the most stable configuration for the Pt dimer is such that the two Pt atoms occupy the neighboring BA site on the BN nanotube, as shown in Fig. 6(a). The average binding energy between the Pt dimer and the $\mathrm{BN}$ nanotube is about $-0.75 \mathrm{eV}$ per Pt atom, less than that of a single Pt atom at the BA site. The bond lengths of Pt-B and $\mathrm{Pt}-\mathrm{N}$ are larger than those shown in Fig. 1(b), indicating that the internal interaction between the two Pt atoms due to the formation of dimer weakens the Pt-BN interaction. The cal- culated band structures show that the band gap of the pristine $\mathrm{BN}$ tube is reduced to $1.38 \mathrm{eV}$ and that two unoccupied impurity states along with several occupied impurity states are induced within the band gap of the pristine tube.

For the Pt-dimer-doped BN nanotube, up to four hydrogen molecules can be chemically adsorbed per supercell, as described in Fig. 6(b). The average $\mathrm{H}-\mathrm{H}$ bond length is $0.82 \AA$ and $\mathrm{H}-\mathrm{Pt}$ bond length is $1.96 \AA$. The average adsorption energy is about $0.31 \mathrm{eV}$ per $\mathrm{H}_{2}$, less than that of a single $\mathrm{H}_{2}$ adsorbed on a single $\mathrm{Pt}$ atom, indicating that the formation of the Pt dimer also weakens the interaction between the $\mathrm{H}_{2}$ and the doped BN nanotube. Again, this adsorption behavior may be explained based on the charge-transfer mechanism discussed above. In fact, the average charge transfer from the $\mathrm{Pt}$ dimer to the $\mathrm{BN}$ nanotube is only $0.097 e$ per $\mathrm{Pt}$ atom, slightly less than that $(0.12 e$ per $\mathrm{Pt}$ atom $)$ from a single $\mathrm{Pt}$ atom to the $\mathrm{BN}$ nanotube.

On the basis of the calculation results, the maximum number of hydrogen molecules chemisorbed on the Pt-doped $\mathrm{BN}$ nanotube can be determined. It appears that an optimal Pt-doped system is such that every BA site of the BN nanotube is occupied by a Pt atom, on which two hydrogen molecules are chemically adsorbed. In reality, however, it is conceivable that several Pt atoms may form a compact cluster on the surface of the $\mathrm{BN}$ nanotube or that the Pt-doped $\mathrm{BN}$ nanotubes may be in rope form. In both cases, the overall amount of chemisorbed $\mathrm{H}_{2}$ can be reduced. As far as the hydrogen storage is concerned, another shortcoming is that the atomic mass of the Pt atom is much larger than that of B or $\mathrm{N}$ atom. This also reduces the hydrogen storage capacity (weight percent) of the Pt-doped BN nanotube. On the positive side, the adsorption and desorption energies of hydrogen on the Pt-doped BN nanotube are typically in the range of a few tenths of $\mathrm{eV}$, a nice range in which the storage and release of hydrogen can be realized at ambient temperature and pressure. To further improve the hydrogen storage capacity of the BN nanotube, it will be desirable that the metal dopants have lighter mass and can cover the surface of the BN nanotube uniformly. This topic will be studied in future work.

Finally, we performed additional calculations using the scalar relativistic density-functional method. With including the relativistic effect of $\mathrm{Pt}$, the interaction between the $\mathrm{Pt}$ atom and BNNT becomes stronger (with a binding energy of $1.80 \mathrm{eV}$ ). The adsorption energy of hydrogen on Pt/BNNT also increases substantially. As such, the third hydrogen molecule can be chemisorbed on Pt. Without including the relativistic effect the third hydrogen molecule is only physisorbed on Pt as shown above. In any case, the upper limit of the number of hydrogen molecules can be adsorbed on a single Pt atom (either chemically or physically) is still three.

\section{CONCLUSION}

We have presented DFT calculation results of hydrogen adsorption on the $\mathrm{BN}$ nanotube doped with a $\mathrm{Pt}$ atom or a $\mathrm{Pt}$ dimer (per supercell). The Pt atom tends to occupy the BA site of the BN nanotube. The doping of the Pt atom induces one unoccupied and five occupied impurity states within the 
band gap. The first hydrogen molecule can be chemically adsorbed on the Pt atom without crossing any energy barrier, while the second hydrogen molecule can be chemically adsorbed on the Pt atom by crossing a small energy barrier. Up to two hydrogen molecules can be chemically adsorbed on a single Pt atom supported by the BN nanotube, for which the average adsorption energy is $-0.365 \mathrm{eV}$. With adsorption of a hydrogen molecule, the interaction between the $\mathrm{Pt}$ atom and the $\mathrm{BN}$ tube is weakened. When two Pt atoms are doped on the $\mathrm{BN}$ tube, the formation of the Pt dimer weakens the $\mathrm{Pt}-\mathrm{BN}$ interaction and lowers the adsorption energy of hydrogen molecule on the Pt dimer. Finally, our calculations offer an explanation to the observed increased percentage of chemical adsorption of hydrogen molecules on the metaldoped $\mathrm{BN}$ nanotubes.

\section{ACKNOWLEDGMENTS}

This work is supported by grants from the DOE (DEFG02-04ER46164), the Nebraska Research Initiative, and by John Simon Guggenheim Foundation and the Research Computing Facility at University of Nebraska-Lincoln.

\footnotetext{
${ }^{1}$ S. Iijima, Nature (London) 354, 56 (1991).

${ }^{2}$ A. C. Dillon, K. M. Jones, T. A. Bekkedahl, C. H. Kiang, D. S. Bethune, and M. J. Heben, Nature (London) 386, 377 (1997).

${ }^{3}$ C. Liu, Y. Y. Fan, M. Liu, H. T. Cong, H. M. Cheng, and M. S. Dresselhaus, Science 286, 1127 (1999).

${ }^{4}$ P. Chen, X. Wu, J. Lin, and K. L. Tan, Science 285, 91 (1999).

${ }^{5}$ C. Zandonella, Nature (London) 410, 734 (2001).

${ }^{6}$ R. Saito, M. Fujita, G. Dresselhaus, and M. S. Dresselhaus, Phys. Rev. B 46, 1804 (1992).
}

${ }^{7}$ A. Rubio, J. L. Corkill, and M. L. Cohen, Phys. Rev. B 49, 5081 (1994).

${ }^{8}$ E. Bengu and L. D. Marks, Phys. Rev. Lett. 86, 2385 (2001).

${ }^{9}$ H. J. Xiang, J. L. Yang, J. G. Hou, and Q. S. Zhu, Phys. Rev. B 68, 035427 (2003).

${ }^{10}$ A. Loiseau, F. Willaime, N. Demoncy, G. Hug, and H. Pascard, Phys. Rev. Lett. 76, 4737 (1996).

${ }^{11}$ R. Z. Ma, Y. Bando, H. W. Zhu, T. Sato, C. Xu, and D. H. Wu, J. Am. Chem. Soc. 124, 7672 (2002).

${ }^{12}$ C. C. Tang, Y. Bando, X. X. Ding, S. R. Qi, and D. Golberg, J. Am. Chem. Soc. 124, 14550 (2002).

${ }^{13}$ X. J. Wu, J. L. Yang, J. G. Hou, and Q. S. Zhu, Phys. Rev. B 69, 153411 (2004).

${ }^{14}$ X. J. Wu, J. L. Yang, J. G. Hou, and Q. S. Zhu, J. Chem. Phys. 121, 8481 (2004).

${ }^{15}$ S.-H. Jhi and Y.-K. Kwon, Phys. Rev. B 69, 245407 (2004).

${ }^{16}$ S. S. Han, J. K. Kang, H. M. Lee, A. C. T. van Duin, and W. A. Goddard III, J. Chem. Phys. 123, 114704 (2005).

${ }^{17}$ X. J. Wu, J. L. Yang, J. G. Hou, and Q. S. Zhu, J. Chem. Phys. 124, 54706 (2006).

${ }^{18}$ Q. Sun, Q. Wang, and P. Jena, Nano Lett. 5, 1273 (2005).

${ }^{19}$ Y. F. Zhao, Y.-H. Kim, A. C. Dillon, M. J. Heben, and S. B. Zhang, Phys. Rev. Lett. 94, 155504 (2005).

${ }^{20}$ T. Yildirim and S. Ciraci, Phys. Rev. Lett. 94, 175501 (2005).

${ }^{21}$ Q. Sun, Q. Wang, P. Jena, and Y. Kawazoe, J. Am. Chem. Soc. 72, 14582 (2005).

${ }^{22}$ S. Dag, Y. Ozturk, S. Ciraci, and T. Yildirim, Phys. Rev. B 72, 155404 (2005).

${ }^{23}$ J. Niu, B. K. Rao, and P. Jena, Phys. Rev. Lett. 68, 2277 (1992).

${ }^{24}$ B. Delley, J. Chem. Phys. 92, 508 (1990); 113, 7756 (2000); DMol3, Accelrys Software Inc., San Diego, CA, 2006.

${ }^{25}$ J. P. Perdew, K. Burke, and M. Ernzerhof, Phys. Rev. Lett. 77, 3865 (1996).

${ }^{26}$ H. J. Monkhorst and J. D. Pack, Phys. Rev. B 13, 5188 (1976).

${ }^{27}$ G. Henkelman and H. Jósson, J. Chem. Phys. 13, 9978 (2000); R. A. Olsen, G. J. Kroes, G. Henkelman, A. Arnaldsson, and H. Jósson, ibid. 121, 9776 (2004) 